\title{
DEVELOPMENT OF A HEMI-SPHERICAL WIDEBAND ANTENNA ARRAY FOR BREAST CANCER IMAGING
}

\author{
I. J. Craddock ${ }^{(1)}$, A. Preece ${ }^{(1)}$, J. Leendertz ${ }^{(1)}$, M. Klemm ${ }^{(1)}$, R. Nilavalan ${ }^{(2)}$ and R. Benjamin ${ }^{(3)}$ \\ ${ }^{(1)}$ University of Bristol, Merchant Venturers Building, Woodland Road, Bristol. BS8 1UB, UK. \\ Email:ian.craddock@bristol.ac.uk \\ ${ }^{(2)}$ Brunel University,UK. Email: Rajagopal.Nilavalan@brunel.ac.uk \\ ${ }^{(2)} 13$ Bellhouse Walk, Kingsweston, Bristol, UK.
}

\begin{abstract}
Using similar techniques to Ground Penetrating Radars, microwave detection of breast tumours is a potential non-ionising and non-invasive alternative to traditional body-imaging techniques.

In order to develop an imaging system, the team at Bristol have been working on a number of antenna array prototypes, based around a stacked-patch element starting with simple pairs of elements and progressing to fully populated planar arrays.

As the system moves closer to human subject trials, a curved breast phantom has been developed along with an approximately hemi-spherical conformal array. This contribution presents, for the first time, details of the conformal array design and initial results from this unique experimental imaging system.
\end{abstract}

\section{INTRODUCTION AND BACKGROUND}

Breast cancer is the most common cancer in women. Xray mammography is currently the most effective detection technique, however it suffers from a relatively high missed- and false-detection rates, involves uncomfortable compression of the breast and also entails exposure to ionizing radiation.

Microwave detection of breast tumours is a potential non-ionising alternative being investigated by a number of groups. In these microwave-based systems, in a similar fashion to Ground Penetrating Radars, microwaves are transmitted from an antenna or antenna array, and the received signals, which contain reflections from tumours, are recorded and analysed.

A pre-requisite for all of these systems is a suitable antenna array. Initial work concentrated on developing a simple but low-profile and wide-band antenna that would cover the $4-10 \mathrm{GHz}$ frequency range. Through extensive FDTD simulation a stacked patch element was designed that broadly meets the design criteria [1]. The stacked patch design was initially employed in a mechanically-scanned system that used stepper motors to move a pair of antennas over a $4 \times 4$ grid of element locations. More recently this arrangement was replaced by the planar stacked-patch array shown in Figure 1.

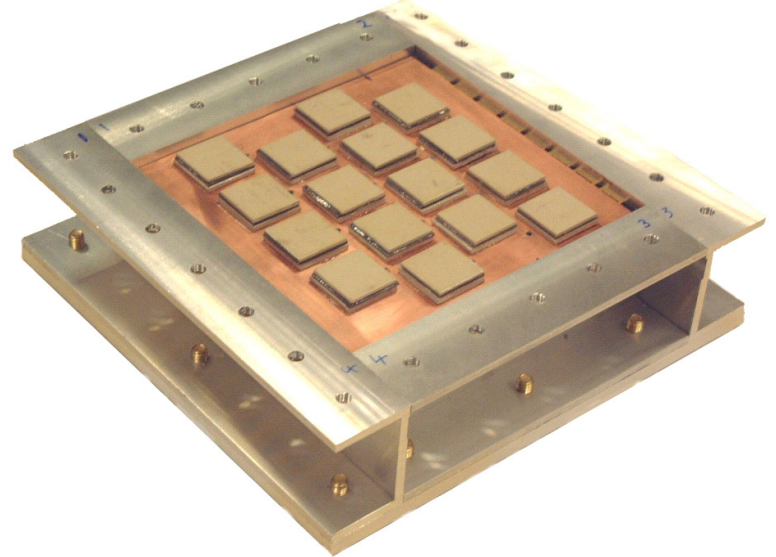

Figure 1: Planar stacked-patch array

Both the mechanically-scanned and fully-populated arrays gave promising results using a "flat" breast phantom consisting of a planar skin phantom and a volume of lossy breast-like phantom medium [2].

The planar geometry is very convenient for array manufacture (the planar array is microstrip-fed on its reverse face) and, indeed, also for the phantom construction. It is difficult to imagine the breast being in uniform contact with such a structure, however, and so in the quest for greater experimental realism, a curved breast phantom has recently been developed along with an approximately hemi-spherical conformal array.

\section{CONFORMAL ARRAY DESIGN}

Given the effort in designing a constructing a conformal array, the intention from the outset was to design not only an array for laboratory use on a realistic, curved phantom, but also one that would serve as an initial clinical prototype.

Approximately 20 female volunteers came forward from the University and the fit between their breasts and 
various plastic spherical sections was assessed with them lying in a prone position - the prone (face-down) position being felt to offer the best chance of the breast forming a gently and uniformly-curved shape.

Following this assessment, the dimensions of the array were input into a 3D CAD model, along with the antenna elements and all supporting metalwork. A view of the CAD model is shown in Figure 2, the array being formed around approximately the lower third of a $78 \mathrm{~mm}$-radius sphere.

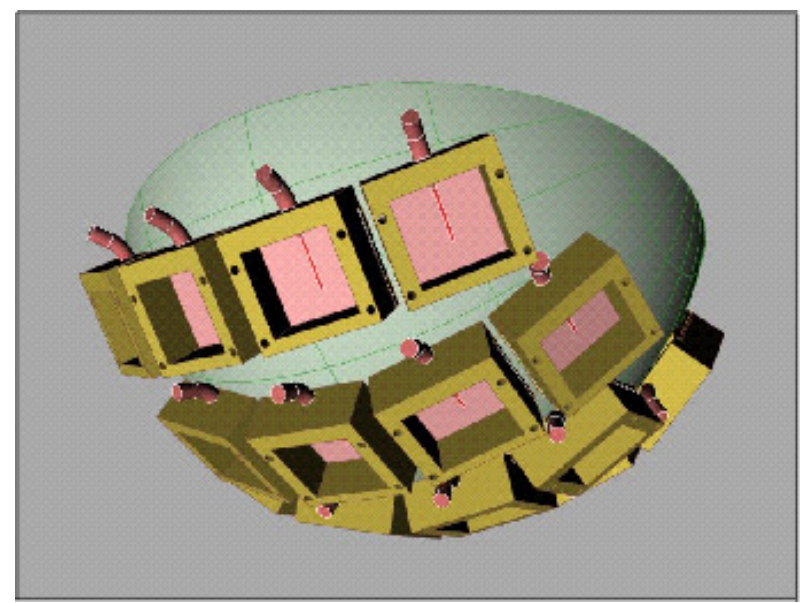

Figure 2: CAD model of conformal array elements

As will be seen in Figure 2, each element is positioned tangentially to the spherical surface. The microstrip feed to the reverse of each element is retained, and clearly visible, however due to the curved construction a transition to semi-rigid coaxial cable is required. The rear of each element is screened (the "lid" being removed in Figure 2 for clarity), unlike the configuration of Figure 1.

The staggered arrangement of elements seen in Figure 1 is retained, this giving - barely - enough clearance for the cables and connectors, which pass between the elements of adjacent rows. The use of the 3D CAD modeling was found to be vital, given the tight geometrical clearances and the difficulty in foreseeing, in a curved geometry, potential clashes between cables, connectors, antennas, screens and supporting metalwork. Even in Figure 2 it may be seen that the right-most feed on the second row (numbered from the top) will not clear the adjacent element - and this required a small modification to the feed arrangement for that individual element.

The partly-constructed array is shown in Figure 3. Two of the antennas in this photograph have had their superstrate glued in place - on the others the uppermost patch is clearly visible.

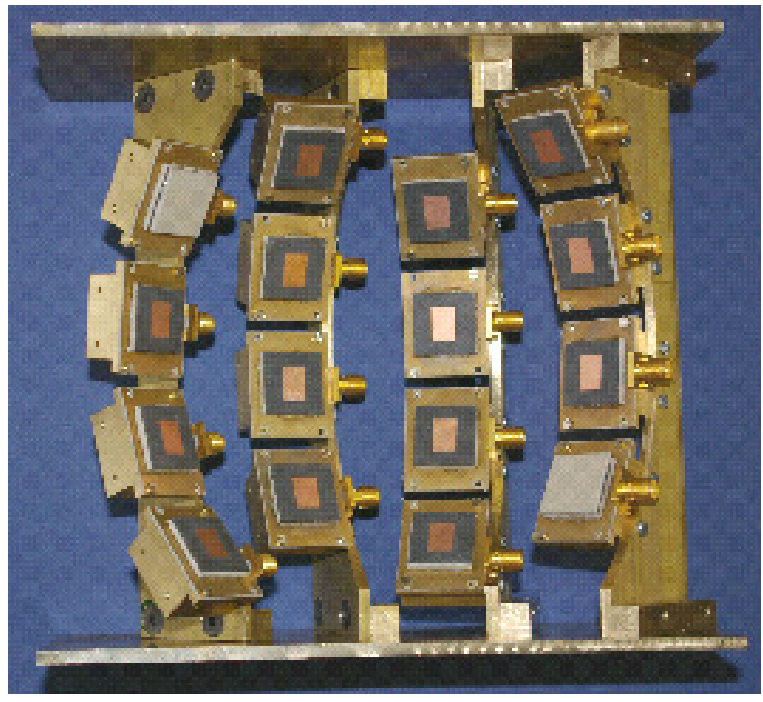

Figure 3: Partly-constructed array

For laboratory tests the array stands-off $20 \mathrm{~mm}$ from a skin phantom and submerged in a tank of liquid breastfat equivalent medium [2]. Figure 4 shows the array with the skin phantom in place.

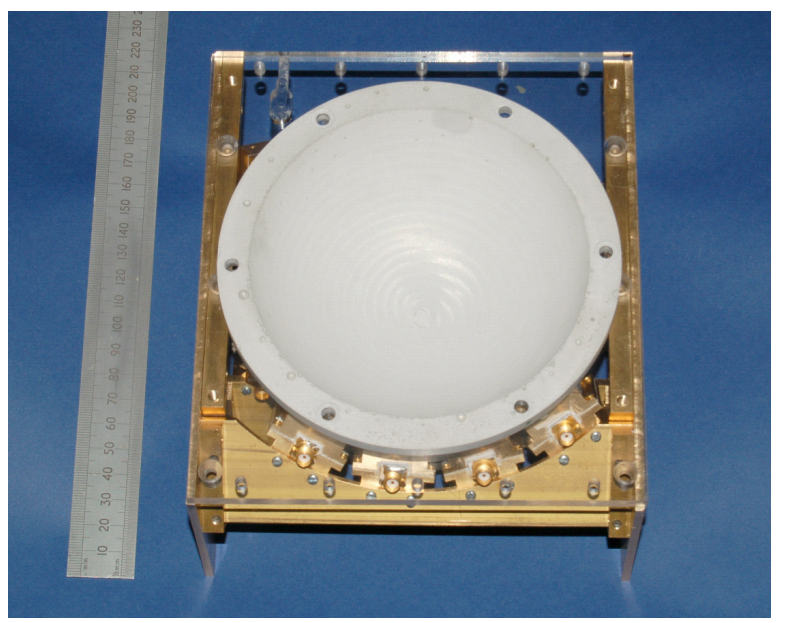

Figure 4: Complete array and skin phantom

The array is connected with semi-rigid coaxial cable to a network of electromechanical switches, previously developed for the planar system. This selects in turn, all possible pairs of elements within the array and connects them to an Agilent network analyser that performs the radar measurement using a frequency-sweep to synthesize time-domain pulsed data. The unused ports of the array (i.e. those not forming the currently-selected pair) are terminated in $50 \mathrm{Ohm}$ loads.

Figure 5 shows the fully operational laboratory system the RF switching between the array and the network 
analyser is beneath the blue metal platform, the array itself is submerged and hence not visible, although the cabling may be seen.

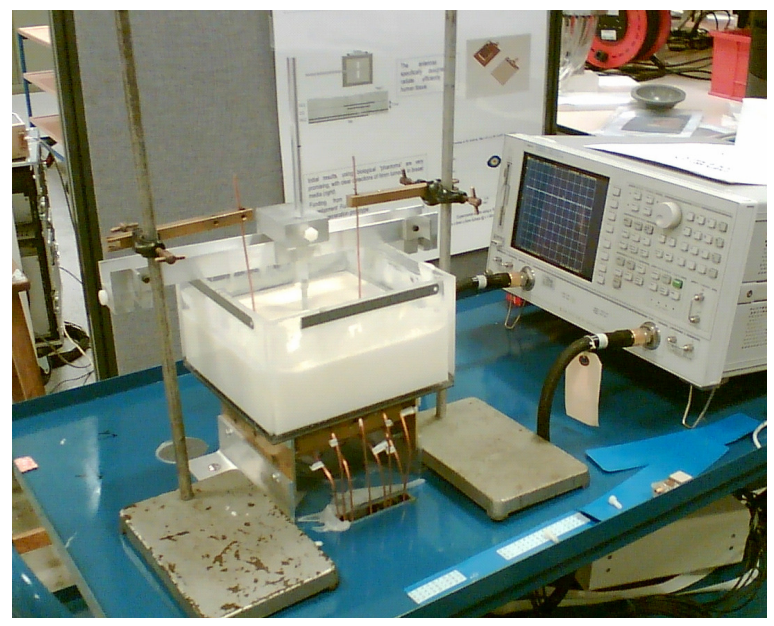

Figure 5: Phantom-based experimental set-up

\section{ARRAY CHARACTERISATION}

Figure 6 presents the input response for the antenna, measured immersed in the liquid medium, this being approximately as expected from previous modelling and measurement, showing a reasonable input match from 4.3 to $9.7 \mathrm{GHz}$. The quality of the match is not quite as good as simulations had predicted, however this is largely attributable to constructional imperfections and is not a particular problem in practice.

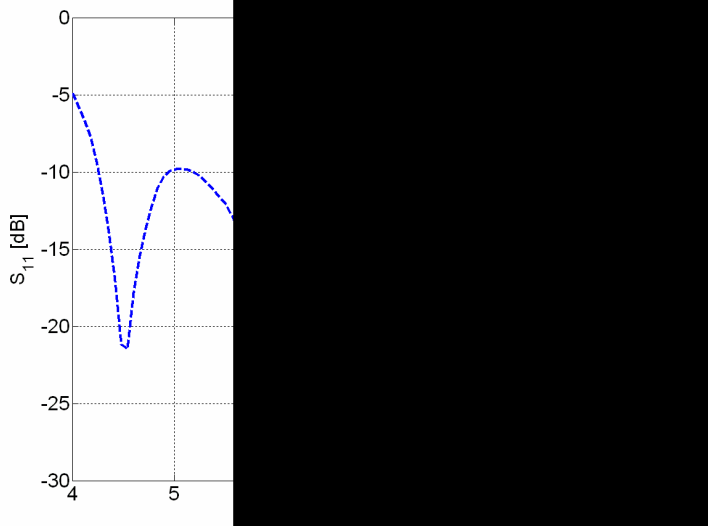

Figure 6: Antenna input response.

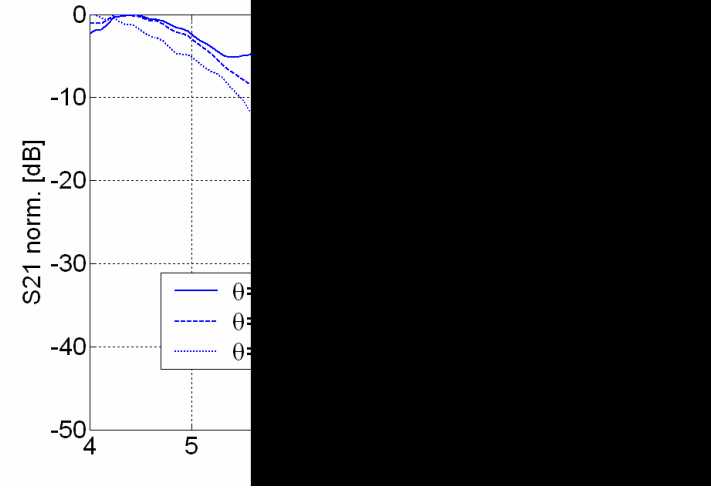

Figure 7: Measured transmission (normalised)

Figure 7 presents the coupling between two antennas, horizontally separated by $10 \mathrm{~cm}$, through the liquid breast equivalent medium (no skin). Although a traditional radiation pattern measurement is impractical, the directivity of the antenna pattern manifests itself very clearly.

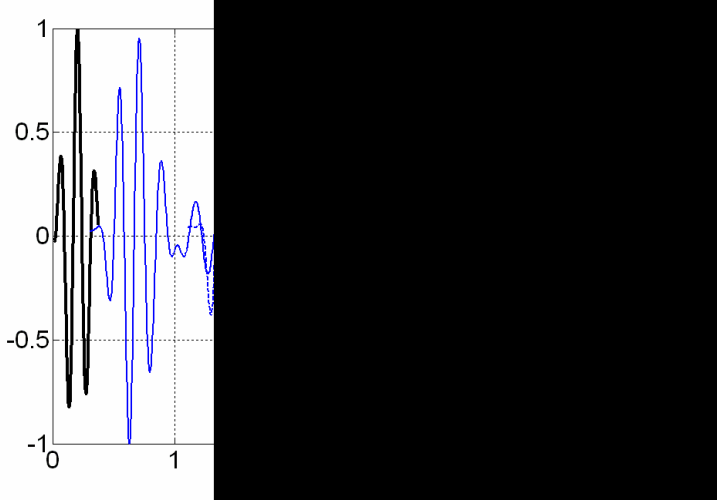

Figure 8: Time domain received pulses

All the subsequent processing of the radar measurement is carried out in the time-domain (see Section 4) by performing an inverse FFT on the frequency domain data, weighted by the spectrum of a hypothetical transmit pulse. Figure 7 shows the assumed transmit pulse, along with the received signal waveforms with different angles between the transmit and receive antennas. Gradual widening of the pulse is seen at offboresight directions, largely due to the frequencydependence of the antenna's radiation pattern (e.g. higher directivity at higher frequencies).

\section{IMAGING RESULTS}

The simplest test of the functionality of the array for imaging purposes is to image a large (phantom) tumour suspended via an index-matched stand within the imaging medium. For this initial test there is no 'skin' 
and mutual coupling between the elements is simply cancelled out by performing a background measurement with the tumour removed and subtracting this from the data. Clearly these are not practical techniques for a clinical system, and considerable effort is being devoted to developing signal-processing that will render this unnecessary.

With 16 antennas in the array, there are $16(16-1) / 2$ unique pairs of antennas in the array, hence 120 signals are recorded.

The recorded data is then synthetically focussed at any point of interest in the volume by time-aligning the signals $y_{i}(t)$, using the estimated propagation time $T_{i}$ from the transmit antenna $A$ to the receive antenna $B$ via the point of interest $C$. The return from $C$ is then computed by integrating the data over a window corresponding to the transmit pulse width $\tau$ :

$$
V=\int_{0}^{\tau}\left(\sum_{i=1}^{N(N-1) / 2} w_{i} y_{i}\left(t-T_{i}\right)\right)^{2} d t
$$

- where $w_{i}$ are weighting factors that are applied to compensate for differences in the predicted attenuation between the round-trip paths (depending on the depth of point $C$ and the spacing from antenna $A$ to antenna $B$ ).
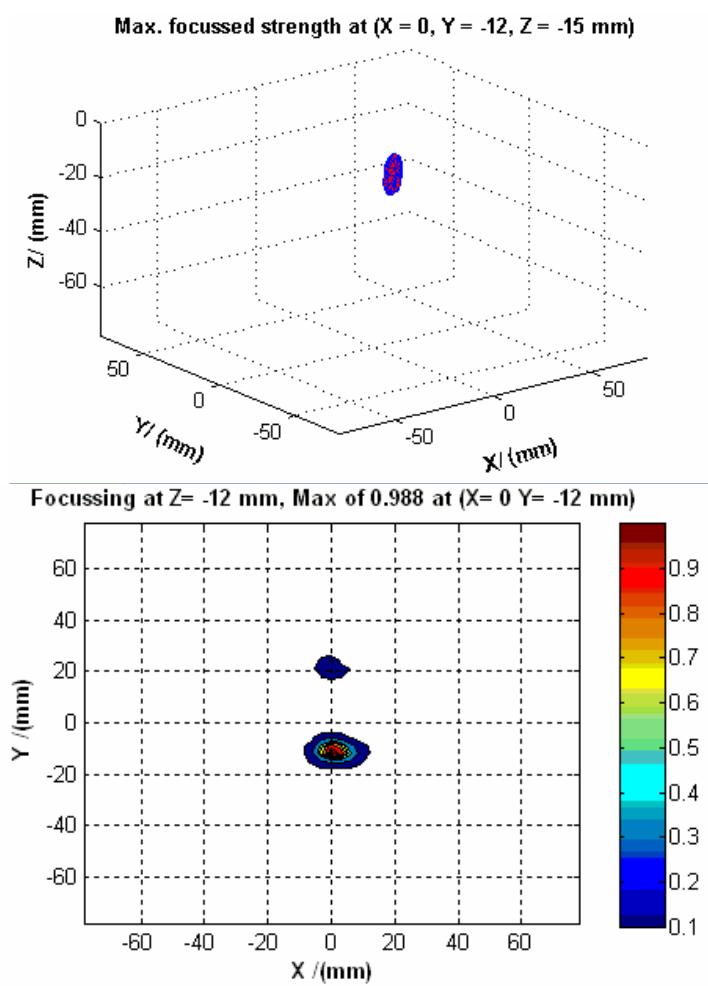

Figure 9: Focused images (3D upper, 2D lower) of a tumour located at $\mathrm{x}=0, \mathrm{y}=0, \mathrm{z}=-10 \mathrm{~mm}$.
The signal processing approach of Equation (1) is similar in essence to other time-shifting algorithms [3, 4], however, in utilising all possible transmit/receive combinations in the array, it differs from these other methods, and the subsequently increased number of observations will offer additional opportunities for clutter rejection.

Figure 9 shows the generated image using equation (1) for every point of the volume of the phantom breast, displayed on a linear colour scale.

The $x, y, z$ co-ordinate origin is the centre of the notional sphere that the array sits upon, hence $x=y=0$, $z=-78 \mathrm{~mm}$ is a point at the centre of the face of the curved array, which is looking "upwards" into the phantom breast.

The tumour is $1 \mathrm{~cm}$ in diameter and positioned at $x=y=0$ and $z=-10 \mathrm{~mm}$. Detection is clear and unambiguous apart from a $10 \mathrm{~mm}$ positional shift along the $y$-axis.

These preliminary results suggest that the array is working well, with a clear detection of the target in admittedly ideal circumstances.

\section{CONCLUSIONS}

A 16 element conformal antenna array has been described. The antenna elements populate the inside of a section of a hemisphere, and this geometry being intended to be suitable for clinical application.

The antenna elements are able to radiate a useable pulse (actually a synthesised pulse) over the $4 \mathrm{GHz}$ to $10 \mathrm{GHz}$ range and are reasonably well-matched to their feeds in this band.

Preliminary imaging results are encouraging and more complex investigations are currently under way, using smaller tumours and with the skin in place. Clinical trials are planned for the present year.

\section{REFERENCES}

[1] R. Nilavalan, I. J. Craddock., J. Leendertz, A. Preece, and R. Benjamin, "Wideband Microstrip Patch Antenna Design for Breast Cancer Tumour Detection" to be published IEE Proceedings, Microwaves Antennas and Propagation.

[2] J. Leendertz, A. Preece, R. Nilavalan, I. J. Craddock and R. Benjamin, A liquid phantom medium for microwave breast imaging, 6th International congress of the European Bioelectromagnetics association, Budapest, Hungary, Nov 2003 
[3] S. C. Hagness, A. Tafove and J. E. Bridges, Twodimensional FDTD analysis of a pulsed microwave confocal system for breast cancer detection: Fixed-focus and antenna-array sensors, IEEE Trans. Biomed. Eng., vol. 45, pp. 1470-1479, Dec. 1998.

[4] E. C. Fear and M. A. Stuchly, Microwave detection of breast cancer, IEEE Trans. Microwave Theory and Tech., vol. 48, pp. 1854-1863, Nov. 2000. 